\title{
Perspectives
}

\section{Peanut lectin crystallography and macromolecular structural studies in India}

\author{
M VIJAYAN \\ Molecular Biophysics Unit, Indian Institute of Science, Bangalore 560 012, India
}

(Fax, 91-80-2360 0535; Email,mv@mbu.iisc.ernet.in)

\section{Introduction}

In India, as in the rest of the world, biological macromolecular crystallography is now considered as an integral part of modern biology. This has happened only comparatively recently. The widespread practice of macromolecular crystallography is also a comparatively recent phenomenon in India. The development of the area in the country resulted from a substantially concerted effort. The structure solution of peanut lectin was a landmark event in this effort. The crystallography of peanut lectin, and that of lectins in general, also hold some lessons in terms of interdisciplinary collaboration, synergy with technological advances and perseverance with a worthwhile problem. I shall attempt here to recount the story of peanut lectin crystallography in the overall historical context of the development of macromolecular crystallography in India. I start with an outline of X-ray crystallography and a brief description of biological macromolecular crystallography, in order to make the rest of the account easily intelligible to non-specialist readers. What is presented here is a personalized account based on the relevant events, as they unravelled, from my perspective.

\section{X-ray crystallography: an outline}

Since its advent in the second decade of the last century, following the discovery of diffraction of X-rays by crystals in 1912 by Max von Laue, X-ray crystallography has been the method of choice for elucidating the structure of matter at the atomic and molecular levels. The early development of the area owed much to the father and son team of William Bragg and Lawrence Bragg, who along with von Laue received the Nobel Prize in 1915. Structure analysis using Xray crystallography is much like the formation of an image in ordinary light microscopy, except for a couple of important differences. The wavelength of X-rays is about five thousand times less than that of light. This wavelength is of the same order of magnitude as the periodicity of the arrangement of atoms, ions or molecules in a crystal. Hence, crystals diffract (scatter in specified directions) X-rays giving rise to hundreds, thousands or hundreds of thousands of intensity maxima or "spots", depending upon the size of the molecule the crystal is composed of. In optical microscopy, the light waves scattered by the object are re-combined by the objective lens to produce the image. Much the same way, an image of the structure can be produced by combining the Xray waves scattered by the crystal. However, no X-ray lens is available. Roughly speaking, the reason is that in the case of $\mathrm{X}$-rays, most materials have (i) refractive indices very close to unity, and therefore poor focussing abilities and (ii) very high absorption coefficients. Therefore, the re-combining has to be done by a mathematical device called Fourier synthesis. There is, however, a catch. A wave has an amplitude and a phase angle. A lens combines light waves automatically taking into account the phase relationship among them. In X-ray diffraction, the intensity of a wave is measured photographically or electronically. The square root of intensities gives the amplitudes of the scattered X-ray waves. But information on phase angles is lost in measurement. Therefore the relative phase angles of the X-ray waves have to be determined before employing the Fourier synthesis. This is the 'phase problem' in X-ray crystallography. Many methods have been devised to overcome it.

\section{From structural chemistry to the centre stage of modern biology}

The first structure to be solved using X-ray crystallography, by the Braggs within a couple of years of the discovery of

Keywords. Ligand specificity; quaternary structure; structural biology; technology in science 
X-ray diffraction, was that of sodium chloride. Since then, for a couple of decades the emphasis in the area was on inorganic compounds. In the nineteen-thirties, organic compounds began to receive increasing attention from crystallographers. The crowning glory of organic crystallography was the determination of vitamin $B_{12}$ structure, for which Dorothy Hodgkin was awarded the Nobel Prize in 1964. Today, the analysis of the structures of ordinary inorganic and organic substances has become almost routine. Methods for deriving phase angles in small molecule crystallography are now highly automated. The methods cannot however be generally used to solve the crystal structures of large biological macromolecules like proteins and their assemblies. Thus, although biological macromolecular crystallography originated in 1934 when the legendary J.D. Bernal and Dorothy Hodgkin (then Crowfoot) recorded the X-ray diffraction pattern from the crystals of pepsin, the first structures that were actually 'solved', those of myoglobin and haemoglobin, were obtained only in the late fifties and the early sixties. John Kendrew and Max Perutz were awarded the Nobel Prize for this work in 1961.

While a normal organic molecule is made up of tens of atoms, a typical protein molecule is made up of thousands of atoms. Roughly, the intensities of X-rays diffracted form a crystal are inversely proportional to the cube of the number of atoms in the molecule in the crystal. Furthermore, the power of the mathematical formulae relating the phase angles of scattered X-ray waves decreases as the number of atoms in the molecule increases. Therefore, overcoming the phase problem in macromolecular crystallography appeared to be an impossible task. However, in the fifties, Perutz demonstrated that the phase problem can be solved using what is called the isomorphous replacement method, if derivative crystals are prepared by attaching heavy atoms in a coherent manner to the protein molecules in the crystal. The preparation of such derivatives is facilitated by the very nature of protein or macromolecular crystals. Unlike crystals of normal organic compounds which are close packed, typically about $50 \%$ of a protein crystal is made up of water. Thus, there are large aqueous regions between protein molecules in the crystal. These regions permit the diffusion of heavy atoms or compounds containing them to the protein, without disturbing the crystalline array. That facilitates the preparation of isomorphous heavy atom derivatives. The early protein structure solutions almost invariably employed the isomorphous replacement method which is often used in combination with what is called the anomalous dispersion method. Independent use of the anomalous dispersion method has also now gained currency. In recent decades, the molecular replacement method, which uses structural information on related proteins, is also extensively used.
The number of protein crystal structures solved in the sixties can be counted on one's fingers. The effort gathered momentum in the seventies. Technological advances in modern biology such as recombinant DNA technology and methods for producing monoclonal antibodies, aided the progress of protein crystallography substantially. This was the case particularly in terms of making available of appropriate samples in large enough quantities. Spectacular advances in X-ray technology also propelled forward macromolecular crystallography. The most important of these advances was the development of dedicated synchrotron sources for X-ray work. In a synchrotron, an electron beam is accelerated along a long circular path. The accelerated electrons emit electromagnetic radiation, including in the $\mathrm{X}$-ray range. The X-rays thus produced are usually several orders of magnitude more intense than those generated by conventional laboratory X-ray sources. Further, when using a synchrotron source, it is possible to choose radiation with a precise wavelength. Thus synchrotron radiation is "tunable". This tunability is very useful when employing the anomalous dispersion method for structure determinations. In the late eighties, position sensitive detectors suitable for recording X-ray diffraction data began to be available for routine use. They are essentially electronic films which combine the advantages of photographic films and electronic counters. In the meantime, the revolution in computation also favourably impinged on the pace of progress in the field. All these developments together propelled macromolecular crystallography into a phase of rapid expansion, a phase which still continues. Structures of thousands of proteins have already been determined and macromolecular crystallography is now recognized as the most important component of structural biology. It has also grown into an integral and essential part of modern biology. Much of what we know now on structure-function relationships in biology at the molecular level is derived from macromolecular crystallography. Crystallographers have now begun to deal with organelles such as the ribosome and cellular components. Often in combination with cryoelectron microscopy, X-ray crystallography is beginning to impact cellular biology. At the same time, it is expected to continue to dominate exploration of the structure and interactions of biomolecules, with added emphasis on areas like structurebased drug and vaccine design.

\section{Birth pangs}

India has a long tradition in crystallography. X-ray crystal structure analysis was initiated at the Indian Association for the Cultivation of Science at Kolkata in the nineteenthirties by K Banerjee, who was an associate of C V Raman. G N Ramachandran and S Ramaseshan, both students of Raman after he moved to Bangalore from Kolkata, 
were responsible for laying the foundations for X-ray crystallography at the Indian Institute of Science, Bangalore, in the late forties and fifties. Ramachandran continued his X-ray crystallographic investigations after he moved to the Madras University in the early fifties. The X-ray work on polymorphism and polytypism pioneered by A.R. Verma at Delhi University, Banaras Hindu University and the National Physical Laboratory, New Delhi, from the early fifties through the seventies and beyond, is also noteworthy. India also had a head start in structural biology, then called molecular biophysics, through the outstanding contributions of G.N. Ramachandran and his colleagues from the midfifties onwards. However, despite the early efforts of Ramachandran himself, we started work comparatively late in biological macromolecular crystallography. The funding for research programmes available in India during the sixties and the seventies was inadequate to initiate a serious macromolecular crystallography effort. Insufficient interactions between crystallographers and biochemists also contributed to the early failures.

A few Indians were involved in the early macromolecular crystallographic studies abroad. Among them, I was the first to return to India in early 1971 , after participating in solving the structure of insulin in Dorothy Hodgkin's laboratory at Oxford. I worked during 1971-74 in the Department of Physics of the Indian Institute of Science, the department in which I did my doctoral work in the sixties, and then moved to the Molecular Biophysics Unit (MBU) founded by G N Ramachandran, who re-joined the Institute in 1971. On my return from Oxford, I would have liked nothing better than to initiate macromolecular crystallography in India. In the then prevalent environment, it was impossible to raise funds to procure even the basic X-ray facilities necessary to initiate such work. Efforts were also made to strike collaborations with the biology departments of the Institute for commencing preliminary crystallization experiments. They did not fructify. Therefore, I concentrated on small molecule crystallography with emphasis on supramolecular association of amino acids and peptides and their implications for chemical evolution and the origin of life. I was also involved in studies on non-steroidal antiinflamatory drugs and ionophores. Although the results of these investigations were extremely gratifying, my main concern was with initiating macromolecular crystallography in the country.

\section{Early efforts}

My association with A. Surolia was an important element in the efforts to start macromolecular crystallographic investigations. Surolia was trained as a graduate student of B.K. Bachhawat, the distinguished biochemist and leader of science, at Vellore and he moved along with
Bachhawat to the Indian Institute of Chemical Biology (then Indian Institute of Experimental Medicine), Kolkata in the mid-seventies. He was then a young man interested in pursuing structure-function relationships in proteins. He was introduced to me by my old friend S.K. Podder of the Department o Biochemistry. We met in 1978 and that was the beginning of a fruitful collaboration, primarily on lectins. That continued to this day. Lectins are proteins which specifically bind different carbohydrate structures (Lis and Sharon 1998; Vijayan and Chandra 1999). They were originally found in plants and their best known property is the ability to agglutinate red blood cells. Subsequently, they were found in animals, bacteria and viruses as well. The realization that most of the recognitive processes in biology, especially on the cell surface, are mediated by sugars, led to substantial interest in protein-carbohydrate interactions and thus in lectins. Lectins were beginning to receive increased attention when Surolia and I started our collaborative effort. The first lectin sample that Surolia supplied was that of RCA from Ricinus communis. We worked on it for three years but it could not be crystallized. In 1980-81, my then graduate student Dinakar Salunke initiated crystallization experiments on samples of peanut lectin supplied by Surolia and his student Islam Khan. The protein crystallized in early 1981. Happily, nearly at the same time Surolia was recruited as a faculty member at the Indian Institute of Science, that too in the Molecular Biophysics Unit, which naturally fostered a higher level of synergetic interactions between our two groups.

Peanut lectin is a non-glycosylated homotetrameric protein with a molecular weight of 1,10,000 Daltons. It is specific to galactose at the monosaccharide level. More importantly, it has high affinity for the T-antigenic disaccharide (Thomsen Friendenreich antigen) Gal $\beta 1$ $3 \mathrm{GalNac}$, which expresses on the cell surface at the onset of some types of carcinoma. Preliminary characterization of peanut lectin crystals was carried out using the rudimentary X-ray facilities available at that time at MBU (see figure 2) and the results were published in the Journal of Molecular Biology in 1982 (Salunke et al 1982). That turned out to be the beginning of a major macromolecular crystallography effort in the country.

In the meantime, the Department of Science and Technology (DST) initiated its Thrust Area Programme. In 1983, when S Varadarajan was the Secretary of the Department, the X-ray group at MBU was funded substantially for work in macromolecular crystallography. Resources above the critical level were for the first time available for serious X-ray diffraction efforts. V Sasisekharan, the then chairman of MBU, was a participant in the effort with his interest in fibre diffraction studies on DNA, and his support was of critical importance (figure 1). MRN Murthy had then just joined MBU with his ambitious 


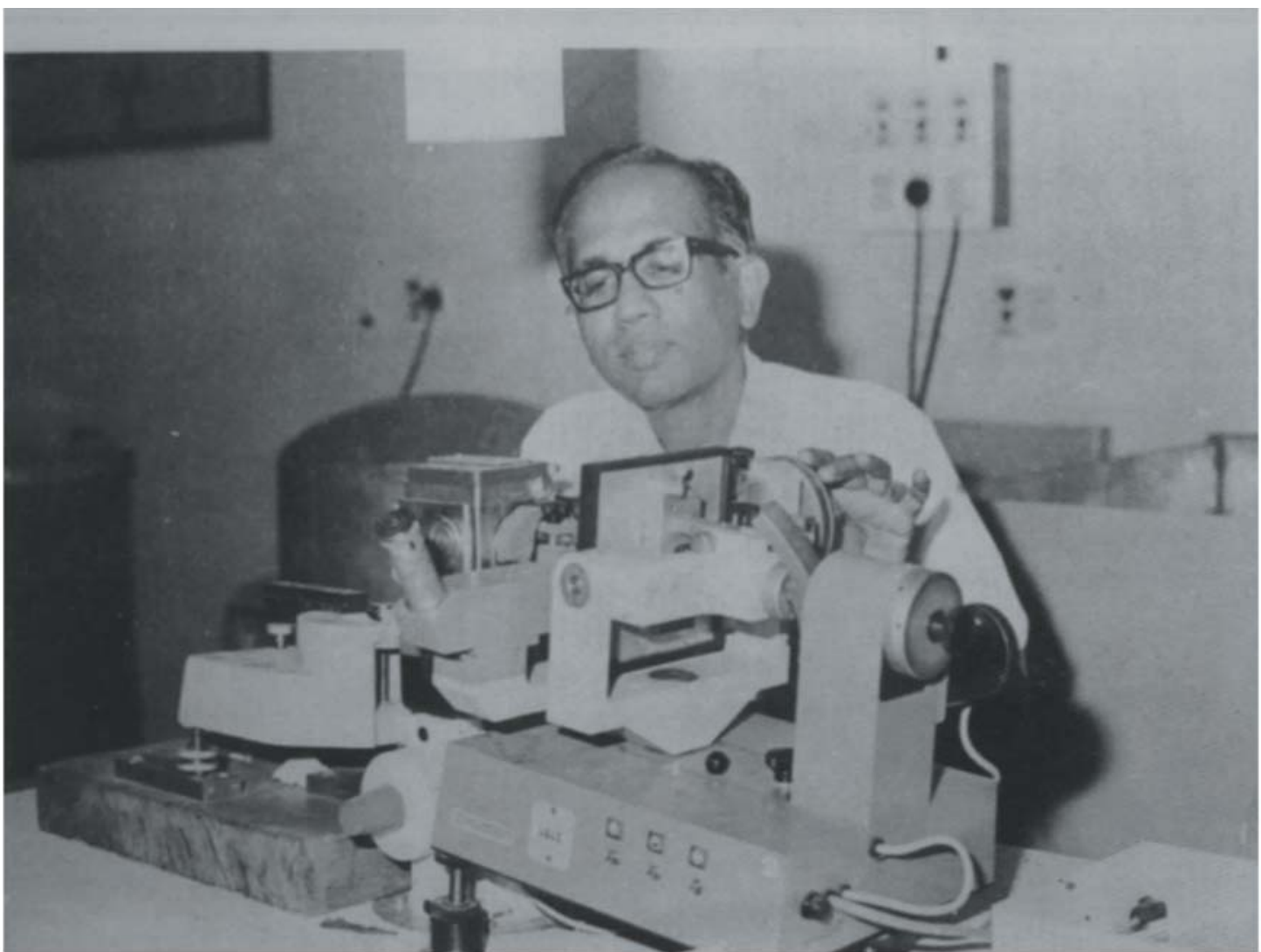

Figure 1. V Sasisekharan working on a precession camera (1984).
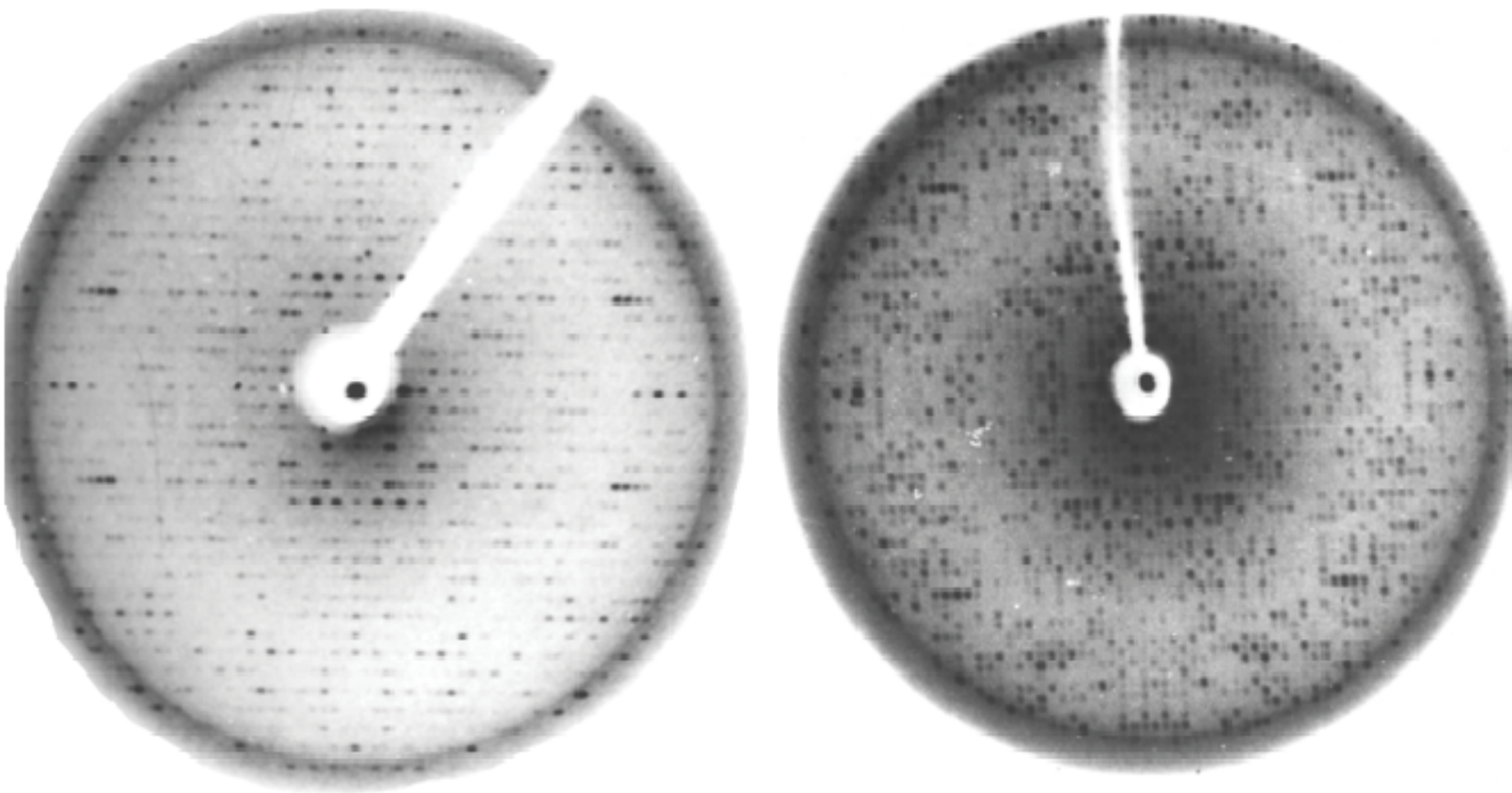

Figure 2. Two original X-ray diffraction photographs of peanut lectin recorded using a precession camera. 
virus crystallography programme. When the support under the Thrust Area Programme was made available, the Bangalore centre had two mandates. One was to build a vibrant macromolecular crystallography centre at the Institute and the other was to function as a national nucleus for the development of the area in the country.

\section{Development of technological competence}

Crystallographic studies on peanut lectin moved in step with the development of the relevant technological competence in the country. In the eighties, internationally the method of choice for X-ray data collection was a combination of synchrotron radiation and oscillation photography. The time required for collecting one data set with such equipment was typically one or more days. In the absence of a synchrotron source as in India, it would take a few weeks or months to collect it using a rotating anode X-ray generator. Clearly, we were at a great disadvantage. However, in the eighties, position sensitive detectors began to replace the photographic camera. By 1990, we could procure an area detector with funds provided under the UGC Centre of Advance Study Programme at MBU and by DST (Eventually that became the basis for a DST supported facility which played a major role in the development of macromolecular crystallography in India). A data set could be collected in a few hours on an area detector using a synchrotron source. The corresponding time is two to few days when the detector is used with a rotating anode generator. Considering the time taken for solving a structure, this time difference is not prohibitively large, although the intensity and tunability of synchrotron radiation cannot be reproduced using an inhouse generator. The availability of an area detector at that stage was invaluable for screening heavy atom derivatives of peanut lectin and collecting data from them and the native crystals.

Laboratories in India had great difficulty in the eighties in procuring computers on account of the declared and undeclared sanctions imposed by the U.S. and other developed countries. For a long time, the Indian Institute of Science was stuck with a DEC 1090 machine supplemented at some stage by a VAX 8800 . although the Government of India had sanctioned funds for a supercomputer on the occasion of the platinum jubilee of the Institute in 1984. It was impossible to deal with a large problem like solving the structure of peanut lectin using a computer of this type when it had to be shared with hundreds of others. In 1990, a CYBER 992 was procured by the Supercomputer Education and Research Centre (SERC) at the Institute. This machine was extensively used for studies on peanut lectin, although it involved re-writing many computer programmes as it used a unique operating system.
Eventually, computation ceased to be a major problem on account of the technological revolution in the field. Again, initially visualization of electron density and models was a serious problem. Electron density maps used to be contoured by hand. Till the mid-eighties crystallographers almost invariably used Evans and Sutherlands graphic machines for their work. However, U.S. had imposed a ban on the supply of these machines to India (but not to China). In the second half of the eighties, this writer took the initiative in contacting the suppliers of the newly developed Silicon Graphics machines. In the early nineties, thanks to funding from the Department of Biotechnology and the active support of N. Seshagiri, the then Director of the National Informatics Centre, New Delhi, a graphics facility could be established with a state of the art Silicon Graphics machine as the centre piece. That facility was of critical importance in our work on peanut lectin. Eventually graphics became common place. Much of the recent work on peanut lectin has been carried out using PCs.

Except for the non-availability of powerful synchrotron sources in the country, we are on par with the rest of the world in the technology of X-ray crystallography. Happily concrete steps are currently on to set up synchrotron facilities in India. Internationally, the emphasis now is on miniaturation and automation. Substantial progress has already been made in rapidly screening a large number of crystallization conditions using very small amounts of precious samples. Technologies for minimizing human intervention in handling crystals have been developed. Efforts are on to produce small laboratory X-ray generators and even small synchrotrons.

\section{An interesting protein}

Although the crystallization of peanut lectin reported in 1982 was followed by several exploratory efforts, the structure could be published only in 1994 (Banerjee et al 1994). By the standards of the eighties, peanut lectin, with a molecular weight of more than a hundred thousand Daltons, was a difficult crystallographic problem to handle, particularly under the conditions then prevalent in India. John Barnabas, a great well-wisher of mine, asked me if I had bitten off more than I could chew. As indicated above, almost at each stage we had to wait for the next stage of development in the relevant technological competence in the country. Furthermore, as we realized later, the nature of the structure of the molecule itself caused difficulty. The structure of tetrameric conconavalin A was well established by the time the structural work on peanut lectin was initiated. Much of our early effort mode use of the molecular replacement method with the help of the known structure of concanavalin A. The efforts failed and the structure could be solved only by the de novo isomorphous replacement 


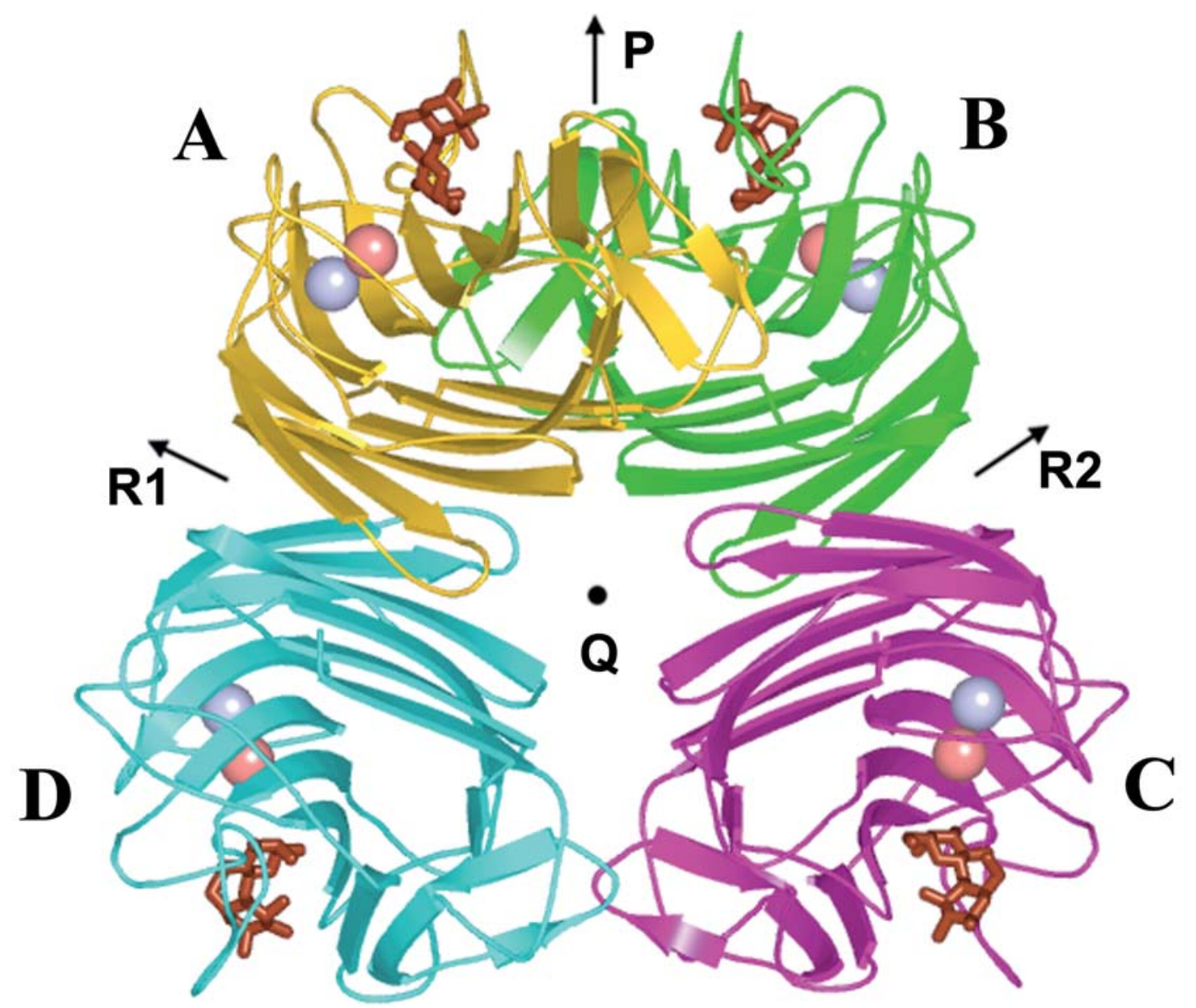

Figure 3. Quaternary structure of peanut lectin. The four subunits are coloured differently. P is a molecular dyad. R1 and R2 are dyads which relate subunits A and D, and B and C, respectively. P, R1 and R2 do not intersect. All the three intersect with $\mathrm{Q}$ at different locations. The bound sugars are in the stick representation. The independent spheres represent metal ions.

method. The reason for the failure of molecular replacement was obvious once the structure was known.

The subunit structures in conconavalin $\mathrm{A}$ and peanut lectin are very similar, but their quaternary structures are entirely different. A well established paradigm pertaining to protein architecture is that multimeric proteins should have a 'closed' structure with point group symmetry. A tetrameric molecule thus should have either $222\left(D_{2}\right)$ or fourfold symmetry. Peanut lectin has neither (figure 3 ). In fact it is the first tetrameric molecule without either symmetry to be characterized. Thus, the structure of peanut lectin showed that open quaternary association without the expected internal symmetry also need to be considered when dealing with oligomeric proteins. The structure of peanut lectin and subsequent studies on related lectins also led to the demonstration that legume lectins are a family of proteins in which small alterations in essentially the same tertiary structure lead to large variations in quaternary association
(Prabu et al 1998,1999; Manoj et al 2000; Kulkarni et al 2004). A detailed X-ray analysis of the crystals of peanut lectin complexed with lactose reported in 1996 led to a full characterization of the legume lectin fold (Banerjee et al 1996). This helped a subsequent detailed analysis of the essential features of proteins containing the legume lectin fold (Chandra et al 2001).

After the mid-nineties, the emphasis in much of the $\mathrm{X}$-ray work on peanut lectin was on the elucidation of the structural basis of carbohydrate specificity using a variety of peanut lectin-sugar complexes (Ravishankar et al 1997,1999; Natchiar et al 2006b). A particularly exciting result was the discovery of the use of water-bridges as a strategy for generating ligand specificity when the lectin binds T-antigen. Such a bridge involves a water molecule which forms a hydrogen bond with the protein a well as the ligand. Differences in the affinity of the lectin for disaccharides with different linkages also could be clearly 
enunciated using the relevant sugar complexes of the lectin. Peanut lectin belongs to a class described as the galactose/ $\mathrm{N}$-acetylgalactosamine binding lectins. Peanut lectin is the only protein belonging to this class which does not bind $\mathrm{N}$-acetylgalactosamine. The reason for this anomaly could be elucidated using the structure, protein engineering and molecular dynamics (Pratap et al 2001). In fact, based on the structure, Surolia and his colleagues could redesign, using site-directed mutagenesis, the combining site of the lectin to alter the carbohydrate specificity of the protein (Adhikari et al 2001). There are few proteins in which the nuances of protein-ligand interactions have been studied as thoroughly as has been done in the case of peanut lectin. The multivalency of the lectin has also been explored (Natchiar et al 2006a). The plasticity and hydration of the lectin has also been studied in considerable detail (Natchiar et al 2004; Natchiar et al 2006b).

Next to conconavalin A, peanut lectin is perhaps the most thoroughly investigated plant lectin. It is certainly the most extensively studied member of the family of lectins conventionally described as galactose $/ \mathrm{N}$-acetylgalactosamine specific. While conconavalin A represents the conventional wisdom on legume lectins, peanut lectin represents departures form it (Natchiar et al 2007). This is particularly evident in relation to quaternary association and sugar specificity.

\section{The aftermath}

In addition to their considerable scientific significance, structural studies on peanut lectin occupy a special place in the development of structural biology in India. The first major successful long term indigenous macromolecular crystallography programme to be launched in the country was that on peanut lectin. The analysis of peanut lectin structure marked the beginning of a large effort on the structural biology of plant lectins, an area in which the Bangalore group is among the international leaders. The effort, which encompassed many non-legume lectins as well (Sankaranarayanan et al 1996; Chandra et al 1999; Pratap et al 2002; Jeyaprakash et al 2004, 2005; Ramachandraiah et al 2003; Singh et al 2005), involved K. Suguna, K. Sekar and Nagasuma Chandra, in addition to Surolia and myself. In the early stages, the development of macromolecular crystallography in India largely paralleled the progress of lectin crystallography. Many students and post doctoral fellows who worked on the structure of lectins, particularly peanut lectin, are among the leaders of structural biology in the country. From humble beginnings a quarter of a century ago, macromolecular crystallography has grown into a strong activity in the country involving close to 20 institutions and nearly twice as many research groups. The systems studied encompass a wide spectrum. Concerted efforts on some of them have had a considerable international impact. A major effort in the country now is on proteins from M. tuberculosis, $P$. falciparum and other microbial pathogens. Focused work is also in progress on disease-related mammalian systems. In addition to advancing the frontiers of knowledge, macromolecular crystallographic investigations in India are also expected to contribute substantially to applications such as structure-based drug and vaccine design. An element of satisfaction at the current state of macromolecular crystallography is perhaps justifiable, although one is conscious that we have to constantly strive to attain greater heights. It is interesting to look back at the beginnings of the effort. Peanut lectin looms large when one does so.

\section{References}

Adhikari P, Bacchawat-Sikder K, Thomas C J, Ravishankar R, Jeyaprakash A A, Sharma V, Vijayan M and Surolia A 2001 Mutational analysis at N41 in peanut agglutinin (PNA): A residue critical for binding of the tumor-associated ThomsenFriedenreich antigen; J. Biol. Chem. 276 40734-40739

Banerjee R, Das K, Ravishankar R, Suguna K, Surolia A and Vijayan M 1996 Conformation, protein-carbohydrate interactions and a novel subunit association in the refined structure of peanut lectin-lactose complex; J. Mol. Biol. 259 281-296

Banerjee R, Mande S C, Ganesh V, Das K, Dhanaraj V, Mahanta S K, Suguna K, Surolia A and Vijayan M 1994 Crystal structure of peanut lectin, a protein with an unusual quaternary structure; Proc. Natl. Acad. Sci. USA 91, 227-231

Chandra N R, Prabu M M, Suguna K and Vijayan M 2001 Structural similarity and functional diversity in proteins containing the legume lectin fold; Protein Eng. 14 857-866

Chandra N R, Ramachandraiah G, Bachhawat K, Dam T K, Surolia A and Vijayan M 1999 Crystal structure of a dimeric mannose specific agglutinin from garlic: Quaternary association and carbohydrate specificity; J. Mol. Biol. 285 $1157-1168$

Jeyaprakash A A, Jayashree G, Mahanta S K, Swaminathan C P, Sekar K, Surolia A and Vijayan M 2005 Structural basis for the energetics of jacalin-sugar interactions: Promiscuity versus specificity; J. Mol. Biol. 347 181-188

Jeyaprakash A A, Srivastav A, Surolia A and Vijayan M 2004 Structural basis for the carbohydrate specificities of artocarpin. Variation in the length of a loop as a strategy for generating ligand specificity; J. Mol. Biol. 338 757-770

Kulkarni K A, Srivastav A, Mitra N, Sharon N, Surolia A, Vijayan M and Suguna K 2004 Effect of glycosylation on the structure of Erythrina corallodendron lectin; PROTEINS: Struct. Funct. Bioinformat. $56821-827$

Lis H and Sharon N 1998 Lectins: carbohydrate-specific proteins that mediate cellular recognition; Chem. Rev. 98 637-674

Manoj N, Srinivas V R, Surolia A and Suguna K 2000 Carbohydrate specificity and salt bridge mediated conformational change in acidic winged bean agglutinin; J. Mol. Biol. 302 1129-1137

Natchiar K S, Jeyaprakash A A, Ramya T N C, Thomas C J, Suguna K, Surolia A and Vijayan M 2004 Structural plasticity of peanut 
lectin: an X-ray analysis involving variation in $\mathrm{pH}$, ligand binding and crystal structure; Acta Crystlogr. D60 211-219

Natchiar K S, Srinivas O, Mitra N, Sagarika Dev, Jayaraman N, Surolia A and Vijayan M 2006a Multivalency in lectins. A crystallographic, modelling and light scattering study involving peanut lectin and a bivalent ligand; Curr. Sci. 90 1230-1237

Natchiar K S, Srinivas O, Mitra N, Surolia A, Jayaraman N and Vijayan M 2006b Structural studies on peanut lectin complexed with disaccharides involving different linkages: further insights into the structure and interactions of the lectin; Acta Crystalogr. D62 1413-1421

Natchiar K S, Suguna K, Surolia A and Vijayan M 2007 Peanut agglutinin, a lectin with an unusual quaternary structure and interesting ligand binding properties; Cryst. Rev. 13 1-26

Prabu M M, Sankaranarayanan R, Puri K D, Sharma V, Surolia A, Vijayan M and Suguna K 1998 Carbohydrate specificity and quaternary association in basic winged bean lectin; J. Mol. Biol. $276787-796$

Prabu M M, Suguna K and Vijayan M 1999 Variability in quaternary association of proteins with the same tertiary fold. A case study and rationalisation involving legume lectins; Proteins: Struct. Funct. Genet. 35 58-69

Pratap J V, Bradbrook G M, Reddy G B, Surolia A, Raffery J, Helliwell J R and Vijayan M 2001 The combination of molecular dynamics with crystallography for elucidating protein-ligand interactions. A case study involving peanut lectin complexes with T-antigen and lactose; Acta Crystalogr D57 1584-1594

Pratap J V, Jeyaprakash A A, Geetha Rani P, Sekar K, Surolia A and Vijayan M 2002 Crystal structure of artocarpin, a Moraceae lectin with mannose specificity, and its complex with methyl-
$\alpha$-D-mannose. Implications to the generation of carbohydrate specificity; J. Mol. Biol. 317 237-247

Ramachandraiah G, Chandra N R, Surolia A and Vijayan M 2003 Computational analysis of multivalency of Lectins: Structures of garlic lectin-oligosachharide complexes and their aggregates; Glycobiology 13 765-775

Ravishankar R, Ravindran M, Suguna K, Surolia A and Vijayan M 1997 Crystal structure of the peanut lectin-T-antigen complex. Carbohydrate specificity generated by water bridges; Curr. Sci. 72 855-861

Ravishankar R, Suguna K, Surolia A and Vijayan M 1999 The crystal structures of the complexes of peanut lectin with methyl$ß$-Galactose and $\mathrm{N}$-acetylactosamine and a comparative study of carbohydrate binding in $\mathrm{Gal} / \mathrm{GalNac}$ specific legume lectins; Acta Crystalogy D55 1375-1382

Salunke D M, Islam Khan M, Surolia A and Vijayan M 1982 Crystallization and preliminary X-ray studies of the anti-T lectin from peanut (Arachis hypogaea); J. Mol. Biol. 154 $177-178$

Sankaranarayanan R, Sekar K, Banerjee R, Sharma V, Surolia A and Vijayan M 1996 A novel mode of carbohydrate recognition in jacalin, a Moraceae plant lectin with a $\beta$ prism fold; Nat. Struct. Biol. 3 596-603

Singh D D, Saikrishnan K, Prashant Kumar, Sekar K, Surolia A and Vijayan M 2005 Unusual sugar specificity of banana lectin from Musa paradisiaca and its probable evolutionary origin. Crystallographic and modelling studies; Glycobiology 15 1025-1032

Vijayan M and Chandra N R 1999 Lectins; Curr. Opin. Struct. Biol. $9707-714$

ePublication: 6 August 2007 